\title{
Lazy Functional \\ Meta-CASE Programming
}

\author{
S. Joosten \\ Computer Information Systems department \\ Georgia State University \\ P.O. Box 4015, Atlanta, GA 30302-4015, USA \\ tel. $+1(404) 6514392$, fax $+1(404) 6513842$ \\ $e$-mail sjoosten@gsu.edu
}

\begin{abstract}
This paper proposes the use of a lazy functional programming language, such as Miranda or Haskell, as embedded language in meta-CASE tools. Functional programming saves time and effort, because method engineers write programs on the appropriate level of abstraction, without any concern about the order of computations. As a consequence, method engineers build more reliable tools for application engineers to work with. The argument is exemplified by a metaCASE program, which defines the well known Entity-Relationship modeling technique. Two different semantics are presented to demonstrate typical use: one is the transformation to a relational model and the other is SQL code generation. The full Haskell program to do this is presented, showing the conciseness and the simplicity of that program. This illustrates the type of programming that is done by a method engineer to specify customized modeling techniques.

This paper is written for designers of CASE and meta-CASE tools. The work is also interesting for functional programmers who wish to see an appropriate application of lazy evaluation.
\end{abstract}

\section{INTRODUCTION}

This paper proposes the use of a lazy functional programming language, such as Miranda [Turner, 1985], or Haskell [Hudak et al., 1992, Hudak and Fasel, 1992] as an embedded programming language in meta-CASE tools.

A meta-CASE tool is a software tool in which modeling techniques are specified in order to generate a CASE tool to support that technique. A modeling technique is a graphical notation (i.e. a picture containing nodes and arcs) with semantics attributed to it. Specifying a modeling technique means to define a class of graphs, to specify the visual appearance of each graph, to delimit the modeling technique by imposing restrictions on the class of graphs, to define operations that change the graph (edit operations) and to 
define operations that interpret the graph (e.g. produce views, transform to other graphs, generate code, perform syntactic and semantic checking)

A method engineer uses a meta-CASE tool, such as MAESTRO [Merberth, 1991] or Meta-Edit [Smolander et al., 1991], for the purpose of creating new modeling techniques, adapting existing techniques to specific requirements, or integrating method fragments into a CASE tool that supports a larger part of a given method [Harmsen et al., 1994, Kumar and Welke, 1992]. Method engineers write programs in order to generate a CASE tool for a particular, user defined modeling technique. Their users are application engineers, who construct application models using a CASE tool generated by a method engineer. An application model is any model used (by application engineers) to build end-user applications.

The method model is the object which is manipulated by a method engineer using a metaCASE tool. A meta-CASE tool contains a method base and CASE tool generators. The method base contains ready-to-use method fragments, from which the method engineer construct (assembles) a method model. These fragments represent modeling techniques (such as ER [Elmasri and Navathe, 1989] or data flow [Yourdon, 1989] modeling), project management techniques (e.g. PERT or GANTT charts), process modeling techniques, configuration management techniques, etc. The method base is enriched by a method engineer, in defining new method fragments. A CASE tool generator is used to generate a CASE tool once the method has been constructed. This generator performs a function similar to a code generator in a compiler.

A meta-CASE programming language is the language embedded in a meta-CASE tool. Method engineers therefore have no choice but to use the language provided with the meta-CASE tool. The particular setting of method engineering yields a unique set of requirements for meta-CASE programming languages. Simplicity and correctness of code are important, because the application engineer does not accept a CASE-tool with errors in it. Although execution performance is important, application engineers do not typically engage in large scale computations. They do require, however that the code generated for their applications is efficient. An important requirement is that a method engineer spends little time programming. A method engineer is supposed to spend time supporting application engineers instead of programming his meta-CASE tool. A complete overview of meta-CASE tool requirements is given in [Harmsen et al., 1994].

This paper starts with the motivation to choose a lazy functional programming language for meta-CASE programming. Next, the Entity-Relationship modeling technique is defined in a functional language (section 3), to exemplify defining a method model. Semantics (meaning) is attributed to the method model in the following section, using the same functional language. This is achieved by defining functions that operate on method fragments. So, form (syntax) and meaning (semantics) can be defined in the same formalism. The next section demonstrates these manipulations on an actual ER diagram (an application model). This model is an instance of the method model defined in section 3. It shows how transformations on models can be combined to achieve larger transformations. It also brings the reader back to the "real world" of actual information systems models. The last part, starting with section 6 , contains a discussion, focusing on the role of the programming language in which method models are expressed. The functional language notation is explained in the appendix. 
I want to thank Sjaak Brinkkemper, Frank Harmsen, Rolf Engmann Maarten Fokkinga, and four anonymous reviewers for their comments on this text. Thanks are also due to Bob Rockwell, who inspired me to write this paper.

\section{Motivation}

Two reasons motivate the choice for having a lazy functional meta-CASE programming language. First, it allows programmers to think on the graph level instead of the node level. Second, it annihilates the issue of control flow, i.e. thinking about a computational order to traverse a graph. Both reasons lead to time savings for the method engineer, and an improved quality of the generated CASE tools. This section explains why.

Programming and thinking in entire graphs produces better code than working on the conceptual level of nodes and arcs does. The functional programming literature at large (e.g. [Harrison, 1993, Joosten, 1989]) shows concise and understandable examples of code which are intricate in terms of nodes and arcs. Kashiwagi and Wise study a general way for implementing graph algorithms in a lazy functional language [Kashiwagi and Wise, 1991]. King and Launchbury demonstrate the usefulness of lazy functional languages for graph algorithms with polynomial complexity [King and Launchbury, 1993]. Meta-CASE tools operate on graphs, which represent modeling techniques. Meta-CASE programming involves graph algorithms that edit graphs, define transformations on graphs, and provide interpretations of graphs. Typical algorithms transform one type of graph into another, which corresponds to deriving one method model from another. This is done on the basis of values, contained in both the nodes and arcs of a graph. Besides, modeling techniques have hierarchical decomposition, making the graph algorithms even more complicated. The structure of data type definitions leads directly to the computational structure of operations on those data types [Gibbons, 1995]. This is helpful to meta-CASE programmers, because it allows them to think beyond the level of nodes and arcs, and work in the realm of graphs as a unit. So, it makes sense to try working in a programming language that supports mathematical abstractions [Harrison, 1993]. Following sections of this paper demonstrate how this works out in one particular example.

Lazy evaluation obliterates the need to think about the order of computations when a graph is traversed. This is caused by the proven fact that a lazy evaluator minimizes the number of evaluation steps for any program. The proof [Abramsky, 1990] consists of showing that a lazy evaluator (i.e. normal order with sharing) executes only essential steps in the computation. A consequence is that all non-essential steps are omitted, producing the minimal number of computation steps for the entire evaluation. This has important consequences for programmers. For example, an nonterminating computation means that no alternative computational order produces a result. This excludes a fair amount of possibilities in searching for errors. It equally well reduces the number of opportunities to make errors in the first place, which reduces programming effort. Graph algorithms are reknown for their intricacies, because graph traversal is often difficult to visualize and imagine. Some errors show up in rare circumstances, adding to the overall perception that graph algorithms are difficult. For this reason, researchers tend to accept only those algorithms that are proven to be correct. In view of this difficulty, a lazy evaluator offers important help to method engineers. If execution fails, the method 
engineer does not spend time to find an execution path that works, as is the case in procedural languages such as Prolan [Merberth, 1991], or even in non-lazy functional languages such as ML [Milner et al., 1989] or Scheme [Rees and Clinger (editors.), 86].

The two reasons mentioned support the argument that meta-CASE programming in a lazy functional language is less time consuming and less error prone. In order to answer how much better it works, comparative research needs to be done using tools that differ only with respect to their embedded programming language. A meta-CASE tool in which the language is a variable and in which a lazy functional language is built, is (to the best of my knowledge) not available. Therefore, the argument has to be settled (for the time being) with the next best thing: examples of typical use.

The choice of an example is guided by "typical use" and by the desire to cover appropriate aspects of meta-CASE programming. In order to cover "typical use" within the limits of a paper, we decided to build a (partial) method model of the Entity-Relationship modeling technique [Elmasri and Navathe, 1989]. This choice was guided solely by the familiarity of the subject matter in the reader community. This choice is meant to keep the reader (as much as possible) on familiar ground with respect to the subject matter. In this way, attention is maximally focused to notation rather than to understanding the subject of ER-modeling. The following aspects were identified to be in the demonstration:

1. representing a graph

Since a model is a graph in all modeling techniques, yet one of a different kind in each different technique, the representation of a graph is an issue. The example given in the following section defines a data structure which represents ER diagrams.

2. representing properties

Properties, both of nodes and of arcs, need to be represented to demonstrate that graph semantics can be represented. The example contains entities and relationships that have attributes and links that have a name and cardinalities associated with them.

3. representing semantics

Semantics are represented by functions that operate on the entire graph, so this needs to be included. The demonstration shows a function which generates SQL code, by way of defining the semantics of the graph.

4. representing transformations

A transformation from the ER diagram to a relational model is defined in our example, to illustrate a transformation from one modeling technique to another, and to illustrate the transformation from a modeling technique to a realization in code.

5. composition of operations

A requirement for meta-CASE tools is that primitive operations on models can be combined in order to define more powerful interpretations and transformations.

6. size of code

The full Haskell code is presented to illustrate the amount of code needed and how it is understood. 
Since method models are essentially graphs that describe other graphs, programming in a meta-case tool consists mainly of graph transformations. The choice for modeling the Entity-Relationship modeling technique [Elmasri and Navathe, 1989] is arbitrary in that any modeling technique which is represented as a graph will illustrate the idea. Readers who prefer to see an example of a process modeling technique, rather than a (static) data modeling technique, are referred to [Joosten, 1994], which contains an example of transforming a business process model into Petri-Nets.

\section{A METHOD MODEL FOR ER DIAGRAMS}

In order to demonstrate the use of a functional programming language, it is inevitable to show some code. Since program code makes notoriously difficult reading, some supplemental documentation is provided in three different ways. First, code is explained elaborately where appropriate. Second, many small examples of the effect of using code have been added. Third, appendix A gives a brief introduction to functional programming. The intention is that readers with no knowledge of functional programming can still appreciate the observations and conclusions (sections 6 and 7).

The method model of Entity-Relationship diagrams [Elmasri and Navathe, 1989] is built up by first introducing entities and relationships. Then, links are introduced and these definitions are assembled into ER diagrams. Operations on ER diagrams are not introduced until section 4 .

\subsection{Entities and Relationships}

Entities and relationships are the main objects in an ER diagram. In Elmasri \& Navathe's notation, they are the nodes in the graph. Each entity and every relationship carries a scheme with the name, attribute and key information in it. The following type is introduced:

\section{data $E R=$ ENT Scheme I REL Scheme}

This definition introduces a type ER, together with the two alternative representations it has. An object with value ENT $\mathbf{s}$ is an entity with scheme $\mathbf{s}$, and an object with value REL $s$ is a relationship with scheme $s$. ENT and REL serve as labels to distinguish the two.

The data declaration introduces a new type that is distinct from any other type in the language. The introduction of a new type, as opposed to using an already defined type, has the advantage that programming errors due to mixing representations are signalled by the type checker.

A scheme is a data structure that contains information about the attributes and keys and also the name of the entity or relationship. The following lines are type synonym definitions, which are recognized by the reserved word type.

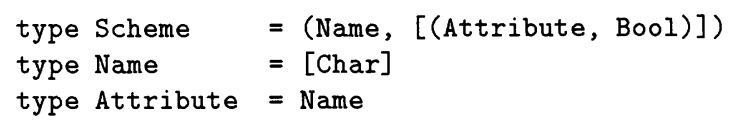


Name is used as a synonym for a list of characters, which is a string. Square brackets mean "list of", so [Char] means a list of characters. Name is used for strings that represent names. Attribute is introduced as a synonym for Name, for the sake of readability.

The type declaration introduces synonyms. Introducing a synonym provides a name for a (possibly more complex) construction of types, and retaining the option of using all the operations that are already defined on that type. The definitions of schemes is not very different from the type definition in procedural languages, where constructs like records and/or arrays would be used. Some languages require upper bounds for the number of characters in a name or the number of attributes in a scheme.

The definition of Scheme involves a tuple (to be recognized by parentheses) that consists of a name (type Name) and a list of attribute/boolean pairs. Here is an example of an object (called testScheme) of the type IverbScheme":

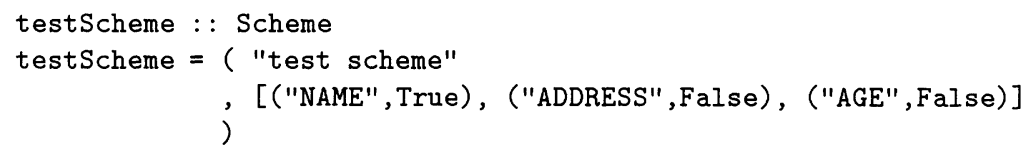

This definition introduces the object testScheme. The double-colon in the first line says that testScheme has type Scheme. The equals-symbol in the second line defines the value of testScheme. As expected, this value consists of a tuple, being a name and a list of attribute-boolean pairs. The boolean that comes with every attribute indicates whether the attribute is a key.

The definition of testScheme uses the property that every object has a direct denotation. If this function were implemented by means of a pointer chain, which is done in languages like Pascal or $\mathrm{C}$, the definition of this example would involve some procedure calls as well, making the code larger and less accessible.

The following auxiliary functions on schemes are defined:

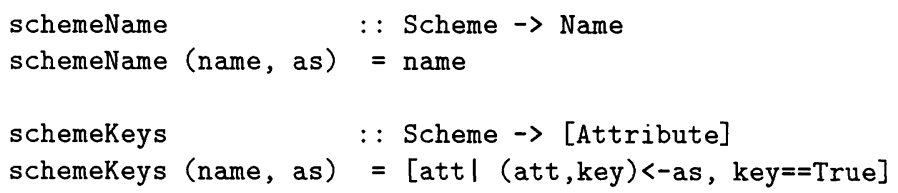

The function schemeName reproduces the name of a scheme, and schemeKeys gives a list of the key attributes. The code of schemeKeys reads: the list of elements called att, in which the pair (att,key) is an element of as and field key equals True.

These definitions illustrate two useful language issues: hiding representations and list comprehensions. Hiding of representations is known as encapsulation in the objectoriented world, and as abstract data types in programming language theory. The functions schemeName and schemeKeys are defined to make the type Scheme independent of its representation. When a programmer decides to change representations, only a few definitions change. It is especially useful for meta-CASE programming, because data structure representations tend to become intricate once a number of different modeling techniques are involved. The list comprehension, used in the definition of schemeKeys, 
appears frequently in places where imperative languages have iteration. Especially termination conditions and loop invariants are a source of errors, which are mostly avoided in the list comprehension.

The following function, showscheme, prints a scheme in ASCII. We use it to demonstrate the dialogue between the user and the computer. The function showscheme shows the previously defined scheme testScheme in a pretty layout.

? showScheme testScheme

test scheme(NAME*, ADDRESS, AGE)

The question mark is the prompt. It is followed (on the same line) by the expression typed in by the user. The result is printed directly underneath.

The code of the function showscheme is presented only for the sake of making a complete presentation.

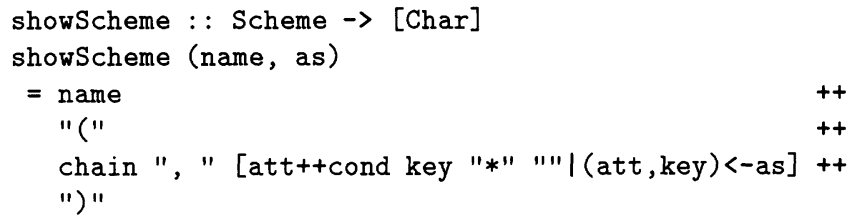

This function prints the name and attributes on one line, Each attribute has affix $*$ if it is a key. The attributes are separated by a comma and a space (", ", by using the function chain) and enclosed in parentheses.

This completes the definition of entities and relationships (i.e. the nodes) in the ER modeling technique.

\subsection{Link}

Now let us observe links, which are represented by lines connecting relationships with entities. A link is the line in an ER diagram that is drawn between an entity and a relationship. Links have a name and a cardinality ratio. The name identifies the role of the link, which is left empty if the role is obvious. The cardinality ratio is also stored in the link.

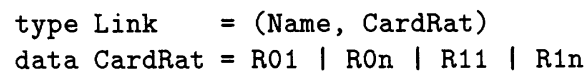

The cardinality ratio of a link is represented by a type CardRat, that can have only four different values: Each value represents a different meaning. Suppose $c$ is the cardinality ratio (type CardRat) of a link between entity $\mathrm{X}$ and relationship $\mathrm{Y}$. The meaning is given by this table:

R01 Each $x$ in X occurs only once or not at all in Y.

R0n There are no restrictions.

R11 Each $x$ in $\mathrm{X}$ occurs precisely once in $\mathrm{Y}$.

$\mathrm{R} 1 \mathrm{n}$ Each $x$ in $\mathrm{X}$ occurs at least once in $\mathrm{Y}$. 
The default value is Ron, which means that there are no restrictions.

The definition of CardRat is an enumerated type, a feature available in many programming languages. Here, the notation is a special case of the data definition encountered in the previous section, so enumerated types do not require a special notation. As opposed to encoding the information (for example as integers) the use of enumerated types results in fewer mistakes, because the compiler signals errors that would otherwise show up at runtime.

A link is shown as an English sentence which states the cardinality restriction imposed by the cardinality ratio. For that purpose the function showCR is defined.

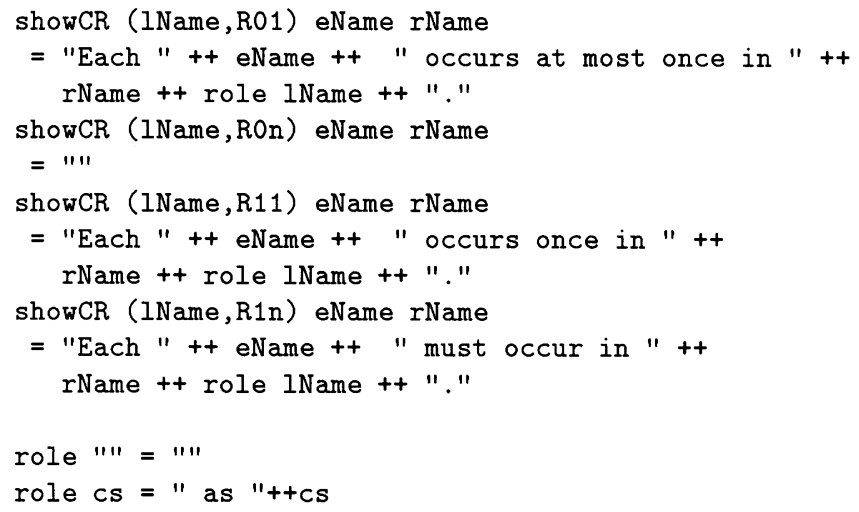

The function role is an auxiliary function, with the sole the purpose of defining showCR. Here is an example of how showCR works.

? showCR ("supervisor", RO1) "EMPLOYEE" "SUPERVISION"

Each EMPLOYEE occurs at most once in SUPERVISION as supervisor.

This function withdraws knowledge from links in a readable form.

This completes the definition of links. The following definitions combine entities, relationships and links to form ER diagrams.

\subsection{ER diagram}

An ER diagram is represented by a graph, with nodes of type ER and links of type Link. Graphically, an ENT node is represented by a rectangle and a REL node by a diamond. Links are represented by lines, connecting an entity and a relationship.

In order to define the ER diagram, an existing type Graph is used. ER diagrams are represented by the type ERdiagram:

type ERdiagram $=$ Graph ER Link

An ER diagram is defined as a graph, with objects of type ER as nodes and objects of type link as arcs (arrows). For ER diagrams, there is nothing else to define. The definition 
of Graph is given in a prelude, so it is not part of the code. Without that, some code defining graphs and operations on graphs would have to be developed.

The definition of ERdiagram is an example of using a data structure (graph), the representation of which is hidden. This type of abstraction is necessary in meta-CASE programming, because graph structures in practice are intricate and therefore error-prone. If a language is used where graphs are defined in terms of pointers to cells, as is the case with a number of procedural programming languages, the definitions are more complicated. Pointer algorithms are notoriously error prone, so it makes sense to avoid their use in meta-CASE programming.

So far, we have discussed new types, synonyms, direct denotation of data objects, absence of pointers, hiding of representations, use of enumerated types, and data abstraction over graphs. The pieces of code presented are tiny illustrations of how these issues help programmers to keep the amount of code low, the level of abstraction high, and the number of mistakes small.

We have introduced the data structure that represents the method model of ER diagrams. Next, operations on ER diagrams are defined to define the meaning of the method model. This is the topic of the next section.

\section{MEANING}

Any method model is useful only if it has meaning, which is achieved by defining operations. These operations provide interpretations of application models, changes to application models, and of course an algebra to create application models. In this section, we illustrate graph transformations that are typical of meta-CASE programming by defining an interpretation of ER diagrams, which is a relational database scheme with integrity constraints. Apart from being an interpretation of ER diagrams, this also demonstrates a transformation from one (well known, prototypical) modeling technique to another. This is an important aspect of meta-CASE programming.

\subsection{Generating attribute vectors}

A relational database scheme consists of a set of attribute vectors and a set of integrity constraints. Generation of the attribute vectors is treated in the first subsection. The next subsection shows how integrity constraints are derived and the third subsection integrates these into relational database schemes.

Every entity or relationship carries its own attributes in a scheme. Semantically, all entities and relationships are mapped on relations in the relational model. Throughout this paper the word 'relation' refers to the relational model, while the word 'relationship' has its meaning within the Entity-Relationship modeling technique.

The choice to map entities and relationships to schemes is typically made by the method engineer. Each entity and every relationship is associated with a scheme that contains all attributes relevant to its meaning. For this purpose the function schemeER is defined, whose effect is illustrated by this example:

? showScheme (schemeER company employee)

EMPLOYEE(Name, Sex, Address, Ssn*, Salary, Bdate) 
The expression "schemeER company employee" yields the scheme that belongs to the node employee within the ER diagram company. Both employee and company are defined in in section 5, which represent a particular ER diagram. This example showed the scheme associated with an entity (employee). Here is another example that shows the scheme associated with a relationship (controls):

? showScheme(schemeER company controls)

CONTROLS (DName*, PName*, DNumber*, PNumber*)

The function showScheme was used to generate pretty output. Without the use of that function, the data structure is printed in full detail (layout added):

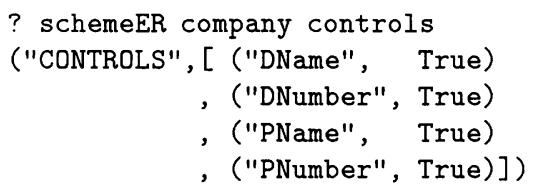

The function schemeER copes with ambiguous attribute names by means of prefixes. Depending on the situation, the attribute name is prefixed with an entity name or a role name. Therefore, an attribute can be renamed if (for every attribute) its name, the entity to which it belongs, and its link name (i.e. role) is known. Due to the disambiguation of names, the code of schemER is more complex than previous examples.

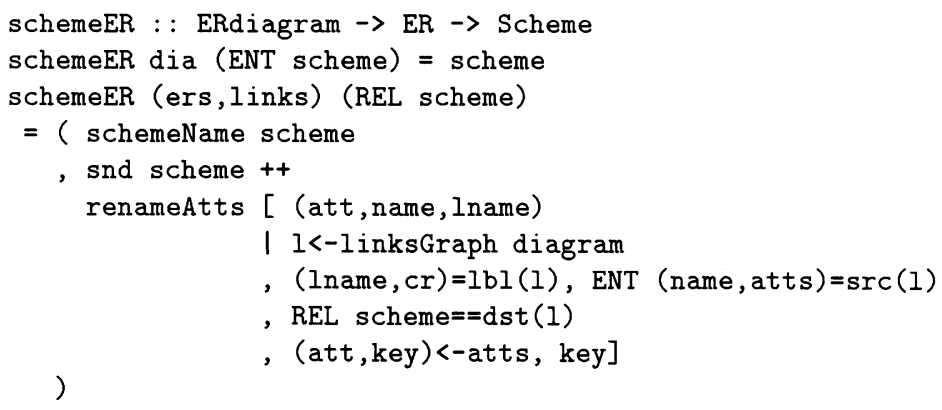

In brief, the function schemeER interprets the graphical elements of ER diagrams and generates a list of attributes (the attribute vector) to be used in a relational database scheme. Entity schemes are copied, whereas the scheme of a relation object is "expanded" by the key-attributes of the adjacent entities.

This is one example which benefits from the fact that control flow is not an issue in a lazily evaluating language implementation. The complexity of the disambiguation problem leads to a complex list comprehension. If the code is written in terms of (imperative) statements, using iteration, selection, and sequence, it becomes even more complicated, because the control flow is no longer as obvious as in simple situations. The opportunities for making mistakes are therefore higher too. Definitions such as schemeER, which are not trivial, are common in meta-CASE programming. 


\subsection{Integrity constraints}

Properties of the ER diagram affect the integrity constraints of the derived database. These constraints limit the possible database instances. The information in the ER diagram makes it possible to derive integrity constraints. A data structure is defined for this purpose.

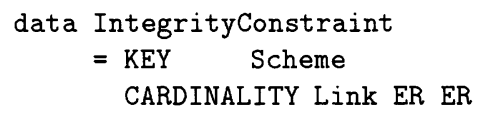

Two types of constraints are introduced. Key constraints specify the candidate keys of each relationship scheme. The cardinality constraint is derived from the cardinality ratio. Referential integrity constraint are not treated here, to keep the volume of the text limited.

In order to print an integrity constraint, the function showIntegrityConstraint is defined. This function is designed to give more or less understandable english sentences for each constraint. For example, the key-constraint associated with the entity employee is printed as:

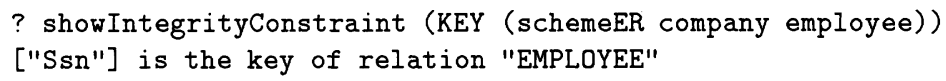

This is how the function showIntegrityConstraint is defined:

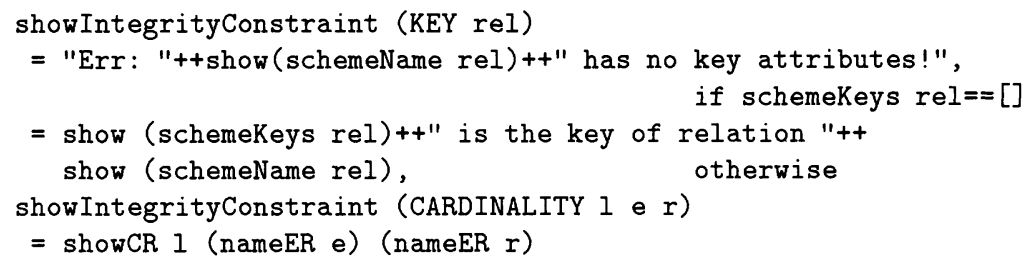

This code uses only language concepts that we used before. Adding concepts to the method model, whether integrity constraints or anything else, leads to a similar use of the language for every new concept introduced. This is the "routine" in which a metaCASE programmer needs to be trained.

Having introduced the concepts of attribute vectors and integrity constraints, we are set to define Relational Database Schemes.

\subsection{Relational Database Scheme}

The ER diagram can be used to derive a Relational Database Scheme (RDS), following the choices made by Elmasri \& Navathe [Elmasri and Navathe, 1989]. A relational database scheme is represented by the type RelDBScheme. It consists of two components: a list of schemes (one for each entity and one for each relationship) and a list of integrity constraints.

type RelDBScheme $=([$ Scheme $]$, [IntegrityConstraint $])$ 
A relational database scheme can be derived from the ER diagram by means of the function rScheme, which is defined by:

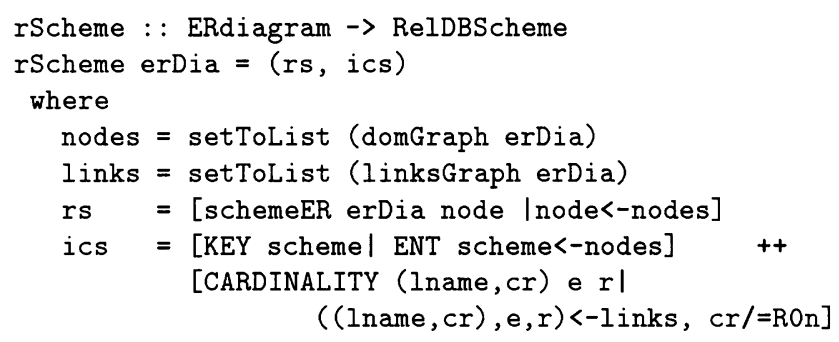

This definition introduces some auxiliaries. The nodes and links of the ER diagram are called nodes and links respectively. The list rs contains the database schemes that correspond to the nodes in the ER diagram. The list ics contains the restrictions. There is a key-restriction for every scheme in the ER diagram and a cardinality restriction for every link. A key restriction that corresponds to an entity contains the same information as the scheme of that entity, so each key restriction is represented by copying the corresponding entity scheme. A similar argument holds for links: the information in the link is used to represent the cardinality restriction of that link. However, links with cardinality Ron have no restriction, so they are filtered out. The database scheme implied by the entire ER diagram is the list of schemes $r$ s together with the restrictions in list ics.

A relational database scheme is a nontrivial interpretation of ER diagrams, especially since cardinalities and integrity constraints are included. Yet, the actual code of rScheme consists of 9 lines only. It is of roughly the same complexity as the code of schemeER, and also roughly as long. Although rScheme is on a higher level of abstraction (mentally) than schemeER, the former is still equally complex for a programmer to understand. The reason is that intelligent use has been made of data abstraction (i.e., hiding representations), and list comprehensions. This is made possible because control flow issues are absent. In an imperative language, or even in a non-lazy functional language, data abstractions can sometimes not be combined freely due to control flow problems, which means that these issues absorb time and energy.

In this section we have seen more complex uses of language constructs and discussed how they help a programmer. We have also introduced the operations that constitute an interpretation of Entity-Relationship diagrams, yielding relational database schemes. This can be seen as the result of a method modeling activity, performed by a method engineer. To generate code, to compute metrics, and to define different views are all uses of a method model that are typically supported by meta-CASE tools. To make transformations from one application model to another and then transform onwards down to a realization requires the ability to cascade computations. A typical example is to transform an ER diagram to a relational database scheme first, and then to generate SQL code from the relational database scheme. The following section demonstrates this as an example of using the method model. 


\section{USING THE METHOD MODEL}

This section shows the transformation to SQL code, as an example to illustrate two points. The first point is that the transformation of an application model to realization is done in a way which resembles the transformation between two application models, by making use of high level abstractions, avoiding control flow issues, and defining a functional transformation concisely. The second point is to illustrate how functional transformations are composed to describe cascaded computations. This composition will in the end be responsible for the practical use and the power of meta-CASE tools, because cascaded transformations create complicated operations that can be used "by pushing one button" in a meta-CASE tool.

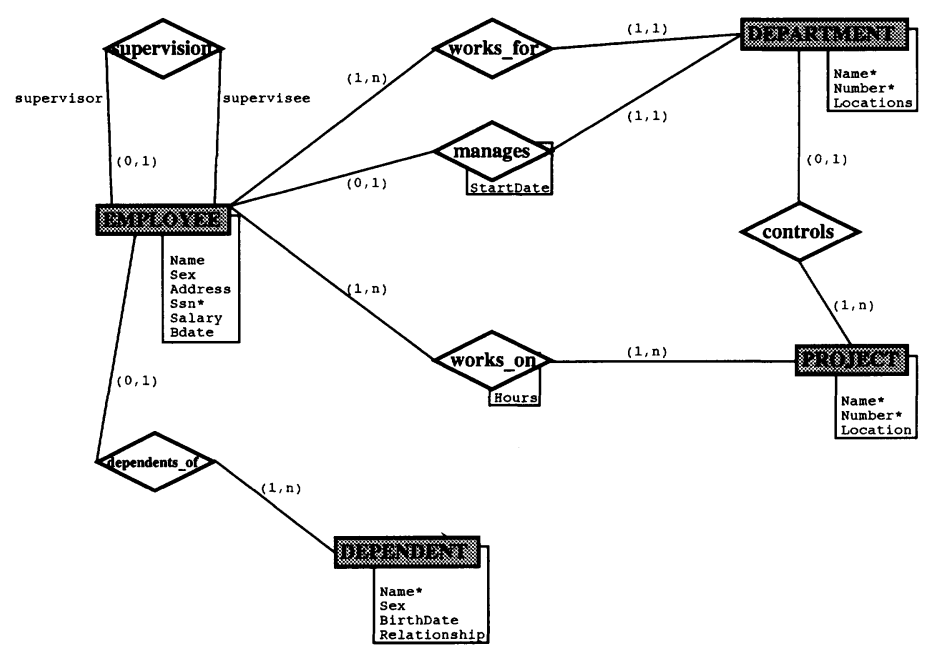

Figure 1 An ER diagram (example, from Elmasri \& Navathe)

To make the demonstration concrete, we show an instance of an ER diagram which is transformed in two subsequent steps. The ER diagram in figure 1 (from fig. 3.13, page 55 in [Elmasri and Navathe, 1989]) is described in terms of the method model in section 3.

Figure 1 has a represention inside the CASE tool (figure 2). This (or similar) code is generated by the CASE tool, derived from the diagram produced by an application engineer. It consists of tabular information representing graphs with attributes on the nodes and arcs. Constraints on the form of the diagram are enforced by the CASE tool. Therefore, syntactical constraints are not needed in the internal representation.

In figure 2, the first definition (company) represents the actual graph. The graph is specified by giving each link together with the two nodes that connect the link. Each link contains a cardinality ratio, which gives restrictions to the number of times an entity may occur in a relation. The (optional) name of the link identifies the role of the entity in the 
relation. The nodes in the graph are either entities or relations, to be distinguished by the labels ENT or REL. Each one of them is defined separately.

For the purpose of this paper the internal representation is written as source code, using the property that even complicated data objects such as an entity-relationship diagram can be denoted directly in the language. The operations defined in section 4 can be used to manipulate the application models. For example, the operation rScheme was defined to transform an entire ER diagram into a relational database scheme. It was used to generate the relational database scheme in figure 3 from the code in figure 2 . We demonstrate how to generate SQL code by giving some definitions (figure 4). We make two assumptions in the code. First, since most SQL implementations have non-standard explicit integrity constraint definitions, or none at all, the code for constraints is omitted (leaving some information unused). Integrity constraints from the ER diagram are enforced in the SQL code by procedural constraints. If the ER diagram is adapted to contain attribute types, better SQL code can of course be generated. The second assumption is that every attribute has the type $\operatorname{VARCHAR}(20)$, because attribute type information is not available in the relational database scheme.

The SQL code in figure 5 was generated by applying the function sqlDBScheme to the relational database scheme of company.

So far, we have seen two transformations. The transformation from entity-relationship diagram to relational database scheme was done by the function rScheme. The transformation from relational database scheme to SQL was done by the function sqlDBScheme. The composition of these functions is defined using the compose operator (the period symbol):

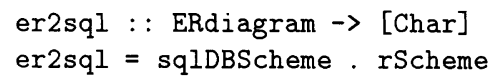

This new function combines both transformations into one new transformations. Composition is an associative operator, which means that an arbitrary number of functions can be cascaded.

\section{OBSERVATIONS}

The elaborated example of the ER modeling technique leads to the following observations:

- Defining a method model of the Entity-Relationship modeling technique is a typical example of method engineering, to be supported by a meta-CASE tool.

- Not treated in this article is the link with the graphics of the meta-CASE tool. Naturally, the embedded programming language contains the graphical primitives needed for the CASE tool.

- The method model of the Entity-Relationship modeling technique is concise. Although ER modeling is not the simplest among modeling techniques, it is modeled within the scope of this single paper. 
- Both the application model (figure 2) and the method model (section 3) and the computations to generate database schemes are described in the same language. All aspects of method modeling can be defined in the same language.

- In the example, data structures are treated 'as a whole'. For example, graphs are used without knowledge of their implementation. This eliminates the need for programming with pointers. As a result, definitions of complicated operations such as rScheme (fewer than 10 lines of code) remain understandable.

- Transformations from one application model to another are within the scope of the language. The operation rScheme is an example of such a transformation. Since all is described in the same language, it is easy to imagine a cascade of transformations. This is useful: the RDB-scheme can be translated further to SQL or any other formalism for that matter. Or, one could decide to insert a normalization algorithm in the cascade of actions.

- Strong typing, which is part of the language, catches type errors at an early stage. This is economical towards a programmer, because new errors that are a consequence of type errors cannot occur. Fundamental errors directly concerning the modeling activity remain. Of course, such errors cannot be detected by any programming system.

\section{CONCLUSIONS}

The observations made in the previous section are related to the requirements identified in the introduction. Let us walk through the list of requirements.

- The level of abstraction in the method model is the level of graphs, schemes, attributes, links, entities and relationships. This is the proper level of abstraction for the method engineer. Since modeling concepts translate directly to types, the effort to reach the proper level of abstraction is minimal.

- Opportunities for mistakes are minimized by:

- defining a data structure as irredundantly as possible; Redundance in data structures is a potential source of errors, because it has consequences for every operator that makes use of the data structure.

- treating a data structure as a whole; For example, a graph is treated as a single object rather than an intricate network of pointers. This eliminates a large source of potential mistakes (with pointers)

- strong typing; A host of trivial checks are unneccessary, because the system is under a strong typing regime.

- absence of side-effects; A program consists of equations, which can safely be interpreted as mathematical equations.

- The tool supports graphs as an abstract data type. 
- Overspecification is avoided mainly because control flow is a non-issue in a (lazy) functional programming language. A program has the same meaning, irrespective of the control flow. This contributes to the conciseness and understandability, and eliminates another source of potential errors.

Considering the use of a method model and the role of a method engineer, the requirements of a programming language for meta-CASE tools match the properties of functional programming. That is: correctness, programmer performance, conciseness and expressiveness are more important whereas requirments on computer performance and access to the hardware are less important. So I conclude that the language is suitable for use in a meta-CASE tool.

The use of one language for the internal representation of both application models and method models offers opportunities for sophisticated functionality, such as the cascading of model transformations.

The following appendix is a superficial introduction to functional programming, intended to assist the reader in understanding the presented code. It is not a short course in functional programming. For that purpose I recommend [Bird and Wadler, 1988]

\section{A A READER'S INTRODUCTION TO FUNCTIONAL PROGRAM- MING}

Functional programming is mainly used for the purpose of specification and prototyping, because it has a high level of abstraction. It is rarely used in cases where efficiency prevails, because low-level features (hardware access) are not available to the programmer.

\section{A.1 Evaluation}

A functional program consists of definitions, which can safely be interpreted as mathematical equations. For example, the definition

$$
f(x)=x * x-2 * x+4
$$

introduces a function $f$, that maps an argument $x$ to the number $x * x-2 * x+4$. So, $f(3)$ equals $3 * 3-2 * 3+4$, which evaluates to 7 . A definition (also called value definition) is recognized by the equals-symbol $(=)$.

The computer serves as a machine, that evaluates expressions. This corresponds to the idea of an ordinary pocket calculator, although the latter works with simpler expressions. The facility to write definitions makes the calculator programmable.

\section{A.2 Types and Values}

All objects in a functional programming language have two components: a type and a value. For example, a variable $\mathrm{x}$ may have type Int and value " 3 ". Consequently, a definition consists of two parts: the type definition and the value definition. The type definition is denoted with a double colon (: :) Example:

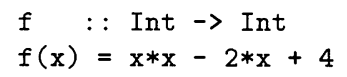


The first line says that $f$ is an object of type Int $\rightarrow$ Int, that is, a function that maps an Int to an Int. The second line defines the value of object $f$, which means that $f$ is the function that maps any $x$ to $x^{2}-2 x+4$. The computer can often derive the type of an object. In that case it is not necessary to specify the type. If the type is specified anyway, the computer uses that information as a check on the derived type. Normal practice is to specify the type to make code more understandable.

There are three forms of type definition. The double colon (:: ) defines the type of an object in the language. The reserved word type introduces a type synonym, which is used for readability purposes. The reserved word data introduces a 'home-made' type. This is used to create data structures suited to particular needs.

A value is given by an expression. A trivial expression, such as 3 , obviously denotes the value 3 . More complicated expressions, such as $3 * 3-2 * 3+4$, require computation to determine the value.

\section{A.3 Notations}

The application of a function to its arguments deserves special attention. The principle is that superfluous parentheses are omitted. So, $f(x)$ is conventionally written as $f x$ by functional programmers. Both have the same meaning.

When more than one argument is involved, the meaning is no longer equivalent. The expression $f(x, y)$ represents the application of a function $f$ to one argument $(x, y)$, which is a tuple containing $x$ and $y$. This is different from $f \mathbf{x} y$ which means the application of a function $f$ to two arguments, $x$ and $y$.

Lists are an important data structure. Square brackets are used to denote lists. Examples:

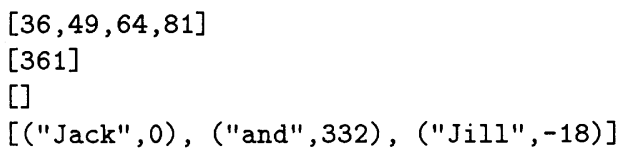

The following notation is frequently used to traverse lists. Here are some examples:

\begin{tabular}{ll} 
expression & result \\
\hline$[x * x \mid x<-[6 \ldots 9]]$ & {$[36,49,64,81]$} \\
{$[y * y \mid y<-[6 \ldots 9]]$} & {$[36,49,64,81]$} \\
{$[$ elem $\mid$ elem<-[0..100], odd (elem) $]$} & {$[1,3,5, \ldots .99]$} \\
{$[a+b \mid a<-[3,30,300], b<-[0,1]]$} & {$[3,4,30,31,300,301]$} \\
{$[a \mid a<-[3,30,300], a<3]$} & {[]}
\end{tabular}

The notation is best understood by pronouncing the square brackets as "the list of", the vertical bar as "in which", and the symbol <- as "traverses" or "is taken from".

Lists can be concatenated by means of the operator ++.

\begin{tabular}{ll} 
expression & result \\
\hline$[2,0]++[4,2,4]$ & {$[2,0,4,2,4]$} \\
"Jack"++"ie" & "Jackie" \\
{[]$++[4,2,4]$} & {$[4,2,4]$}
\end{tabular}




\section{References}

[Abramsky, 1990] Abramsky, S. (1990). The Lazy lambda-calculus, pages 65-117. Addison Wesley.

[Bird and Wadler, 1988] Bird, R. and Wadler, P. (1988). Introduction to Functional Programming. International Series in Computer Science. Prentice Hall, New York.

[Elmasri and Navathe, 1989] Elmasri, R. and Navathe, S. B. (1989). Fundamentals of Database Systems. Addison-Wesley World Student Series. Benjamin/Cummings, Redwood City, CA 94065.

[Gibbons, 1995] Gibbons, J. (1995). An initial-algebra approach to directed acyclic graphs. In Möller, B., editor, Mathematics of Program Construction, number 947 in Lecture Notes in Computer Science, pages 122-138, Berlin. Springer-Verlag.

[Harmsen et al., 1994] Harmsen, F., Brinkkemper, S., and Oei, H. (1994). Situational method engineering for information system projects. In Olle, T. and Stuart, A. V., editors, Proceedings of the IFIP WG8.1 Working Conference CRIS'94, pages 169-194, Amsterdam. North-Holland.

[Harrison, 1993] Harrison, R. (1993). Abstract data types in Standard ML. John Wiley \& Sons, Chichester, England.

[Hudak and Fasel, 1992] Hudak, P. and Fasel, J. H. (1992). A gentle introduction to haskell. ACM SIGPLAN Notices, 27(5).

[Hudak et al., 1992] Hudak, P., Peyton Jones, S. L., and Wadler (editors), P. (1992). Report on the programming language haskell, a non-strict purely functional language (version 1.2). SIGPLAN Notices, 27(3).

[Joosten, 1989] Joosten, S. (1989). The use of functional programming in software development. PhD thesis, University of Twente, dept. of Comp. Sc.

[Joosten, 1994] Joosten, S. (1994) Trigger modelling for workflow analysis. In Proceedings CON '94: Workflow Management, Challenges, Paradigms and Products (Oct. 1994), G. Chroust and A. Benczúr, Eds., Oldenbourg, Wien, München, pp. 236-247.

[Kashiwagi and Wise, 1991] Kashiwagi, Y. and Wise, D. S. (1991). Graph algorithms in a lazy functional programming language. Technical Report 330, Comp. Sci. Dept, Indiana Univ., Bloomington, Indiana.

[King and Launchbury, 1993] King, D. J. and Launchbury, J. (1993). Functional graph algorithms with depth first searc. In Hammond, K. and O'Donnell, J. T., editors, Functional programming, volume II, pages II.1-II.12. Springer-Verlag, Berlin.

[Kumar and Welke, 1992] Kumar, K. and Welke, R. J. (1992). Methodology Engineering: A Proposal for Situation-Specific Methodology Construction, chapter 15, pages 257-269. Series in Information Systems. John Wiley, Chichester. 
[Merberth, 1991] Merberth, G. (1991). Maestro II - das integrierte CASE-System von Softlab. BI Wissenschafsverlag, 3 edition.

[Milner et al., 1989] Milner, R., Tofte, M., and Harper, R. (1989). The Definition of Standard ML. MIT Press, Cambridge, MA.

[Rees and Clinger (editors.), 86] Rees, J. and Clinger (editors.), W. (86). The revised report on the algorithmic language scheme. SIGPLAN Notices, 21(12):37-79.

[Smolander et al., 1991] Smolander, K., Lyytinen, K., Tahvanainen, V.-P., and Marttiin, P. (1991). Metaedit - a flexible graphical environment for methodology modelling. In Andersen, R., Bubenko, J., and Solvberg, A., editors, Proc. Third International Conference on Advanced Information Systems Engineering (CAiSE'91), number 498 in Lecture Notes in Computer Science, Berlin. Springer-Verlag.

[Turner, 1985] Turner, D. A. (1985). Miranda: A non-strict functional language with polymorphic types. In Jouannaud, J.-P., editor, 2nd Functional programming languages and computer architecture, number 201 in Lecture Notes in Computer Science, pages 1-16, Berlin. Springer-Verlag.

[Yourdon, 1989] Yourdon, E. (1989). Modern Structured Analysis. Yourdon Press Computing Series. Yourdon Press, New Jersey. 


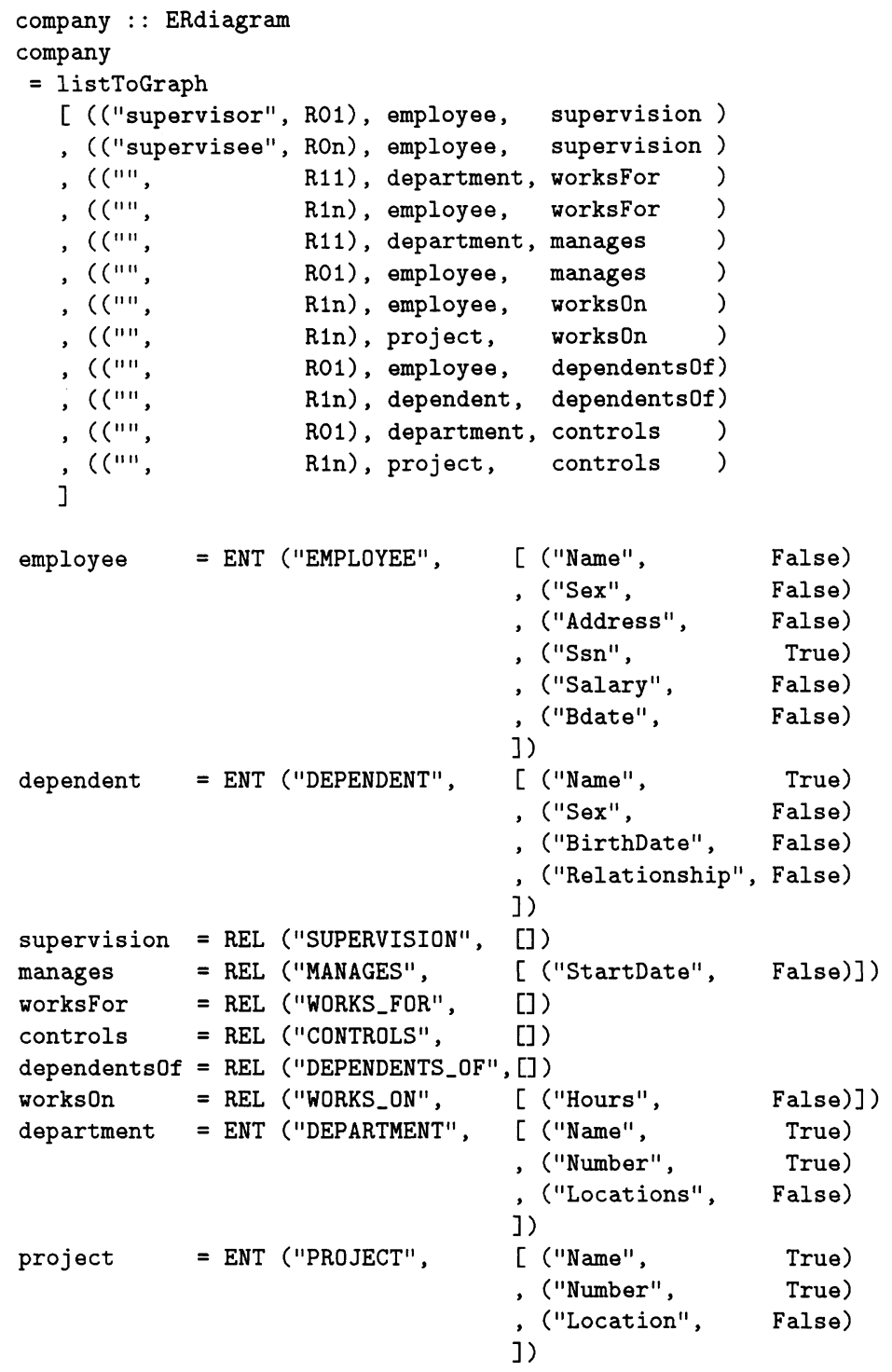

Figure 2 internal representation of ER diagram 


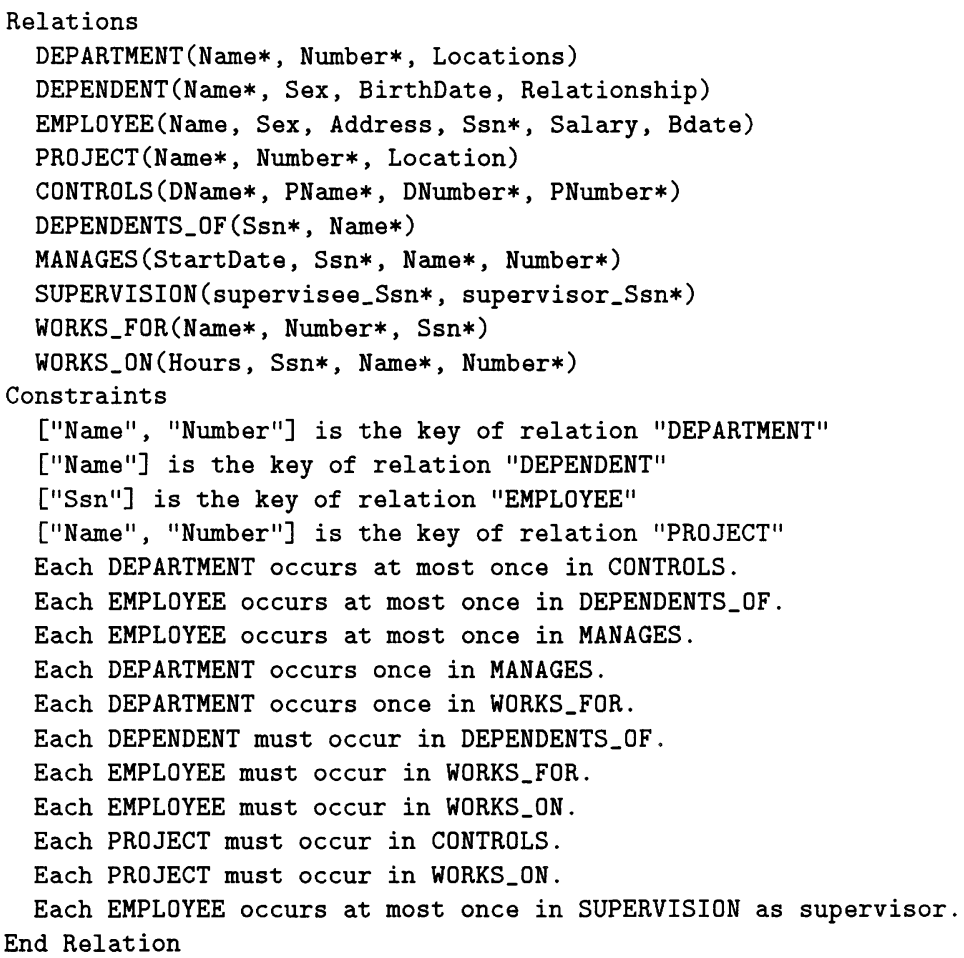

Figure 3 result of operation rScheme 


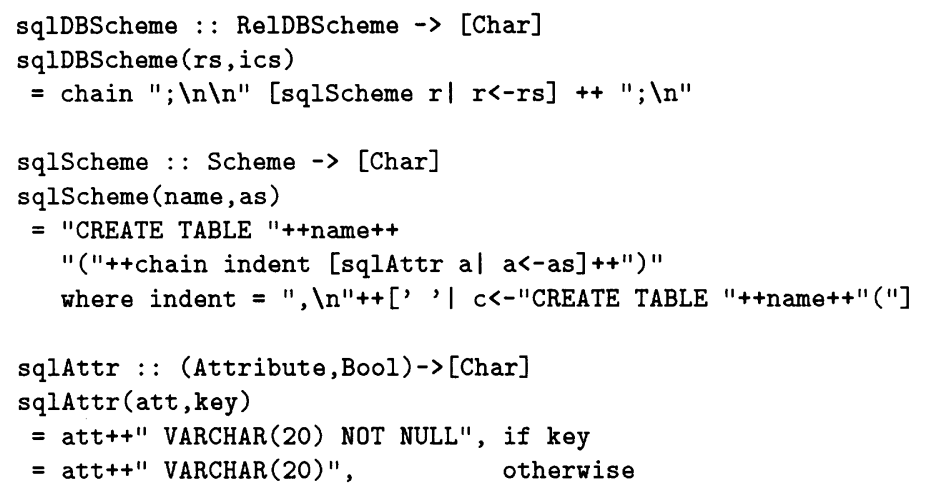

Figure 4 SQL generator 
? sqlDBScheme( $r$ Scheme company)

CREATE TABLE DEPARTMENT(Name VARCHAR(20) NOT NULL, Number VARCHAR(20) NOT NULL, Locations VARCHAR(20));

CREATE TABLE DEPENDENT(Name VARCHAR(20) NOT NULL, Sex VARCHAR(20), BirthDate VARCHAR(20), Relationship VARCHAR(20));

CREATE TABLE EMPLOYEE(Name VARCHAR(20),

Sex VARCHAR (20), Address VARCHAR(20), Ssn VARCHAR(20) NOT NULL, Salary VARCHAR(20), Bdate VARCHAR(20));

CREATE TABLE PROJECT(Name VARCHAR(20) NOT NULL, Number VARCHAR(20) NOT NULL, Location VARCHAR(20));

CREATE TABLE CONTROLS(DName VARCHAR(20) NOT NULL, PName VARCHAR(20) NOT NULL, DNumber VARCHAR(20) NOT NULL, PNumber VARCHAR(20) NOT NULL);

CREATE TABLE DEPENDENTS_OF(Ssn VARCHAR(20) NOT NULL, Name VARCHAR(20) NOT NULL);

CREATE TABLE MANAGES(StartDate VARCHAR(20), Ssn VARCHAR(20) NOT NULL, Name VARCHAR(20) NOT NULL, Number VARCHAR(20) NOT NULL);

CREATE TABLE SUPERVISION(supervisee_Ssn VARCHAR(20) NOT NULL, supervisor_Ssn VARCHAR(20) NOT NULL);

CREATE TABLE WORKS_FOR(Name VARCHAR(20) NOT NULL, Number VARCHAR(20) NOT NULL, Ssn VARCHAR(20) NOT NULL);

CREATE TABLE WORKS_ON(Hours VARCHAR(20), Ssn VARCHAR(20) NOT NULL, Name VARCHAR(20) NOT NULL, Number VARCHAR(20) NOT NULL);

Figure 5 generated SQL code 